\title{
High-Q photonic chip-based temporal phase plates for electron microscopy
}

Armin Feist ${ }^{1}$, Arslan Sajid Raja ${ }^{2}$, Jan-Wilke Henke ${ }^{1}$, Junqiu Liu ${ }^{2}$, Germaine Arend ${ }^{1}$, Guanhao Huang ${ }^{2}$, Fee Jasmin Kappert ${ }^{1}$, Rui Ning Wang ${ }^{2}$, Jiahe Pan $^{2}$, Ofer Kfir ${ }^{1}$, Tobias Kippenberg ${ }^{2}$ and Claus Ropers ${ }^{3}$

${ }^{1} 4$ th Physical Institute - Solids and Nanostructures, University of Göttingen, Göttingen, Germany, United States, ${ }^{2}$ Institute of Physics, Swiss Federal Institute of Technology Lausanne (EPFL), Lausanne, Switzerland, United States, ${ }^{3} 4$ th Physical Institute - Solids and Nanostructures, University of Göttingen, Göttingen, Germany, Goettingen, Niedersachsen, Germany

The active optical shaping of free-electron beams enables a broad range of applications, from efficient free-space acceleration [1] and attosecond bunching of electrons [2-4] to the implementation of laser-driven phase plates [5,6] and beam splitters [7].

Recent experiments on inelastic electron-light scattering (IELS) [8,9] with dielectric microcavities such as microspheres [10] or photonic crystal cavities [11] have demonstrated the potential of using resonant field enhancement for increasing the interaction between electrons and photons. However, bringing IELS to state-of-the-art continuous-beam electron microscopes is challenging, due to the optical transition probabilities normally being far below unity, thus requiring the use of femtosecond high-intensity laser pulses.

In this work, we demonstrate IELS on a CW-pumped $\mathrm{Si}_{3} \mathrm{~N}_{4}$ microresonator with a $Q$-factor of $>10^{6}$, achieving an unprecedented high coupling to a continuous electron beam. The interaction strength between the electrons and the evanescent cavity field is spatially mapped and we discuss the potential application as a versatile temporal phase plate for electrons.

In an ultrafast transmission electron microscope (UTEM) [12], a continuous electron beam passes by a CW pumped $\mathrm{Si}_{3} \mathrm{~N}_{4}$ microresonator (see Fig. 1.a)), which is installed in a custom-designed TEM sample holder. The air-cladded microresonator chips (Fig. 1.b)) are fabricated using the photonic Damascene process [13] and exhibit a linewidth of $\sim 70 \mathrm{MHz}$ (Fig. 1.c)), as well as a free spectral range of $\sim 1 \mathrm{THz}$ for the quasi-TM fundamental mode. The electron beam interacts with the whispering gallery mode, confined in the fiber-coupled photonic chip.

When the CW laser is tuned to a resonance of the cavity, the initially narrow energy distribution is significantly broadened up to about $50 \mathrm{eV}$, with a shape typical for multi-order absorption and emission of photons(Fig. 1.a,d)). We retrieve a spatial map of the electron-light coupling parameter $g$, which exhibits a pronounced modulation along the structure (Fig. 1.e)), due to Ramsey-type interference [14] between the two crossings of the electrons and the resonator ring.

In conclusion, we demonstrate strong inelastic electron scattering off a CW optical field, enabled by high-Qmicroresonators. Hereby, new opportunities arise in electron microscopy, from optical beam structuring with state-of-the-art integrated photonics, to the exploration of free-electron cavity quantum optics[15]. 
b)

c)
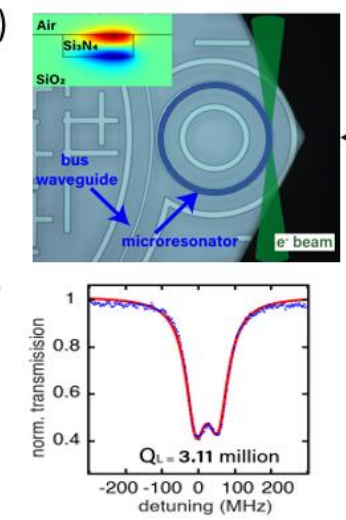

a)

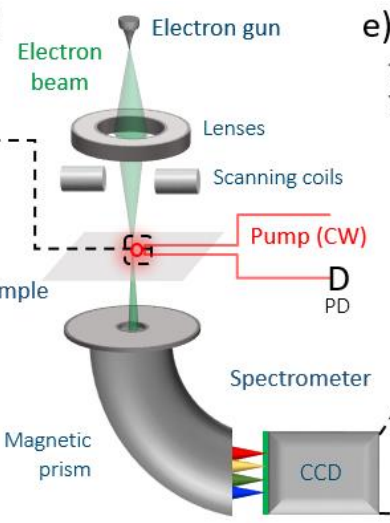

e)
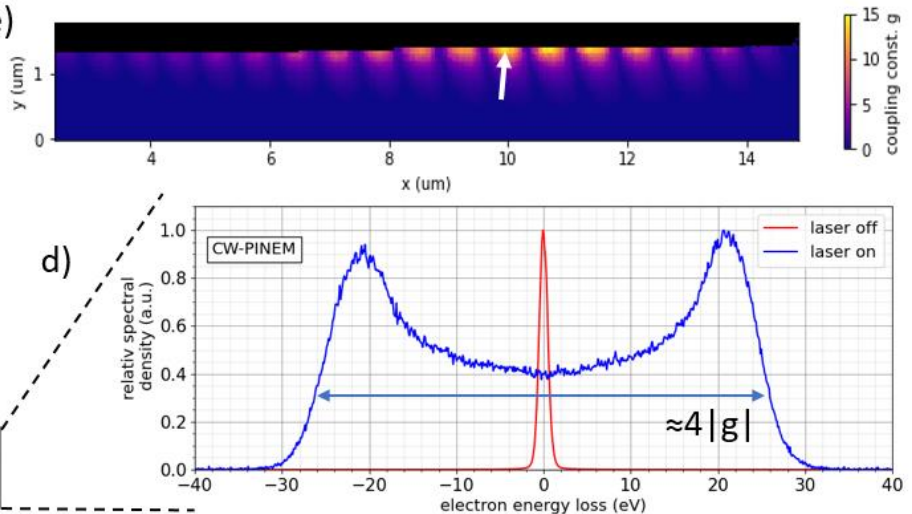

Figure 1. Figure 1. a) Inside of a transmission electron microscope (TEM), an electron beam passes close to a CW-pumped integrated Si3N4 microresonator in aloof geometry (b, SEM image: side view of the chip, Inset: Electric field component parallel to the electron beam.) c) Optical transmission spectrum of a typical microresonator resonance exhibiting a linewidth of $\sim 70 \mathrm{MHz}$ (Q-factor of 3.11-106). d) Electron energy distribution of the transmitted beam recorded with a magnetic-prism spectrometer. Tuning the $\mathrm{CW}$ laser to a cavity resonance, a spectral broadening up to $\sim 50 \mathrm{eV}$ is observed (probing position indicated by the white arrow in e)). e) Spatial map of the electron-light coupling constant $\mathrm{g}$, obtained from scanning the electron beam across the resonator.

\section{References}

[1] R. J. England et al., Rev. Mod. Phys.86, 1337-1389 (2014).

[2] K. E. Priebe et al., Nat. Photonics 11, 793 (2017).

[3] Y. Morimoto and P. Baum, Nat. Phys. 14, 252 (2018).

[4] M. Kozáket al.,Phys. Rev. Lett. 120, 103203 (2018).

[5] O. Schwartz et al. Nat. Methods16, 1016-1020 (2019).

[6] G. M. Vanacoreet al., Nat. Mater. 18, 573-579 (2019).

[7] A. Feist et al.,Phys. Rev. Research 2, 043227 (2020).

[8] B. Barwick, D. J. Flannigan, and A. H. Zewail, Nature 462, 902 (2009).

[9] F. J. García de Abajo, et al., Rev. Mod. Phys. 82, 0034-6861 (2010).

[10] O. Kfir et al., Nature 582, 46-49 (2020).

[11] K. Wang et al., Nature 582, 50-54 (2020).

[12] A. Feist et al., Ultramicroscopy 176, 63 (2017).

[13] J. Liu et al., Optica 5, 1347-1353 (2018).

[14] K. E. Echternkampet al., Nature Physics 12, 1000-1004 (2016)

[15] O. Kfir et al., Phys. Rev. Lett. 123, 10 (2019). 